\title{
Telomerase inhibition by an siRNA directed against hTERT leads to telomere attrition in HT29 cells
}

\author{
PATRÍCIA DE SOUZA NASCIMENTO ${ }^{1,2}$, GILDA ALVES $^{3}$ and WOLFGANG FIEDLER ${ }^{1}$
}

\begin{abstract}
${ }^{1}$ Klinik und Poliklinik f. Innere Medizin I - Sektion Molekulare Gastroenterologische Onkologie, Martin Luther Universität Halle-Wittenberg, Halle, Germany; ${ }^{2}$ Departamento de Genética, Instituto de Biologia, CCS, Universidade Federal do Rio de Janeiro; ${ }^{3}$ Laboratório de Genética Aplicada, Serviço de Hematologia, Instituto Nacional de Câncer, Rio de Janeiro, Brazil
\end{abstract}

Received December 19, 2005; Accepted February 3, 2006

\begin{abstract}
Human telomerase is a ribonucleoprotein complex composed of at least the reverse catalytic transcriptase hTERT and RNA component hTR. The enzyme stabilizes telomere length and thereby contributes to unlimited cell proliferation, i.e. immortality. Reactivation of telomerase activity during carcinogenesis is a common hallmark in most human tumor types. Consequently, telomerase is an attractive molecular target toward which to direct cancer therapeutic agents. RNA interference (RNAi) has been shown to be an effective method for inhibiting the expression of a given gene in human cells by targeting with short duplex RNA (short-interfering RNA or siRNA). Thus, we evaluated the ability of siRNAs to inhibit telomerase activity in the HT29 immortal human colorectal adenocarcinoma cell line as a model for colorectal carcinogenesis. By transient expression of a specific siRNA directed against hTERT, we reduced telomerase activity in the transfected cells. Moreover, telomere lengths were reduced in cells stably expressing this particular RNA sequence, cloned as an shRNA into an eukaryotic expression vector. The cell clone that displayed a telomerasenegative phenotype showed dramatically reduced telomere lengths and stopped proliferation. We observed that the vector was integrated into the cell genome and, despite telomere shortening, cells retained their MSI phenotype. We conclude that we have developed a potent telomerase inhibitor leading to cell death.
\end{abstract}

\section{Introduction}

Human telomerase is a specialized ribonucleoprotein composed at least of two core components; the RNA component, hTR,

Correspondence to: Dr Gilda Alves Brown, Instituto Nacional de Câncer, Serviço Hematologia, Laboratorio de Genética Aplicada, Praça da Cruz Vermelha 23, $6^{0}$ andar, CEP: 20230-130, Rio de Janeira, Brazil

E-mail: gbrown@inca.gov.br

Dr Wolfgang Fiedler, Martin Luther University, KIM I - Molecular Gastroenterological Oncology, Ernst Grube Str. 40, 06120 Halle, Germany

E-mail:wolfiedler@web.de

Key words: colorectal adenocarcinoma, hTERT knockdown, siRNA, telomerase activity and hTERT, the catalytic subunit responsible for reverse transcriptase activity. They jointly maintain the telomere length of the chromosomes, with hTR serving as a template for hTERT activity, which catalyzes the polymerization of telomeric repeats (1). As tumor cells must stabilize telomeres to maintain their proliferation capacity and escape from replicative senescence, the reactivation of telomerase activity and telomere maintenance in association with tumorigenesis is well described for nearly all cellular types, but not in most normal cells (2). While hTR has been detected as ubiquitously present in almost all human cells, hTERT seems to be expressed only in active telomerase cells. Thus, the inhibition of hTERT expression may be a promising therapeutic strategy for selectively blocking telomerase activity in cancer cells, including colorectal cancer, where hTERT mRNA expression is gradually up-regulated during adenoma-carcinoma progression and may be a rate-limiting determinant of telomerase activity during tumor malignancy (3).

Several approaches have been described to successfully block telomerase in human cells by various pharmacological drugs, chemical agents, ribozymes and antisense molecules $(4,5)$. Telomerase inhibition by azidothymidine was previously described in the HT29 colorectal cell line (6). However, a more specific and efficient inhibition system for telomerase is still of interest. A new strategy for specific knockdown of a gene of interest is the RNA interference (RNAi) technique (7). RNAi is a natural post-transcriptional sequence-specific gene regulatory mechanism of the cell that controls gene expression by degrading the corresponding endogenous mRNA. The mediators of that process are small double-stranded RNAs (21 to 23 bp in length), the so-called short interfering RNA (siRNA). siRNAs are processed from larger dsRNAs by the Dicer enzyme and form the RISC complex (RNA-induced silencing complex) that guides the siRNA to the corresponding homologue mRNA and cleaves it at the binding site region (8).

Recently, different approaches to successfully block telomerase activity by variable siRNA techniques have been described for several cell lines, excluding colorectal lines (9-11). Therefore, we were interested in the long-term effects of telomerase inhibition in the p53-deficient HT29 human colon cancer cell line by a potent siRNA targeting hTERT. HT29 is a telomerase-positive colorectal adenocarcinoma cell line showing mutant p53 phenotype (12) but no mismatch repair deficiency (13). For this study, we designed three siRNA 
Table I. siRNA sequences and their respective target regions on hTERT mRNA.

\begin{tabular}{llc}
\hline Name & siRNA sequence ${ }^{\mathrm{a}}$ & hTERT mRNA target region $^{\mathrm{b}}$ \\
\hline siRNA \#1 & 5'-AACCUUCCUCAGGACCCUG-3' & Exons 10/11 \\
siRNA \#2 & 5'-CGUUCCGCAGAGAAAAGAG-3' & Exon 4 \\
siRNA \#3 & 5'CUUGCGGAAGACAGUGGUG3' & Exon 11 \\
\hline
\end{tabular}

${ }^{a}$ The above-indicated sequences correspond to the siRNA sense strand relative to the hTERT cDNA sequence. ${ }^{b}$ The three selected molecules were directed against regions on hTERT mRNA that codify the reverse transcriptase structural motifs.

molecules directed against three different defined regions of the hTERT mRNA. By transient transfection experiments of HT29 cells, we ruled out the best inhibitory one. This particular sequence was cloned as a short hairpin (shRNA) into an eukaryotic expression plasmid, and the long-term consequences were observed again by the transfection of HT29 cells.

We isolated a cell clone with clear down-regulation of expressed hTERT mRNA and decreased telomerase activity. Subsequently, these cells showed dramatic telomere length attrition. Furthermore, the cells stopped proliferation after 22 cell doublings. As shortened telomeres could account for the replicative senescence process, we additionally proved that the cells showed no microsatellite instability (MSI). In conclusion, using our strategy, it is possible to find a potent telomerase inhibitor and observe the consequences of longterm inhibition of telomerase knockdown.

\section{Materials and methods}

Short-interfering RNA (siRNA) design. siRNAs directed against hTERT mRNA were selected using the Qiagen software analysis system, and it was verified, by BLAST search, that there was no homology with another human gene. siRNA \#1-3 sequences directed against hTERT mRNA were selected according to Blast search score and GC content (40-60\%). The sense and antisense strands of the siRNA duplexes were $19 \mathrm{nt}$ with two unpaired thymines (TT) at the 3' overhangs (Table I). The control siRNA sequence (5'GCAAGCUGACCCUGAAGUUCAU-3') named GFP-22 siRNA, directed against the green fluorescence protein (GFP) mRNA, was ordered from Qiagen control library.

Cell culture. The ATCC HT29 colorectal adenocarcinoma cell line was grown in DMEM (Dulbecco's modified Eagle's medium) supplemented with $10 \%$ FCS (fetal calf serum), $4 \mathrm{mM}$ L-gluthamin, $1 \mathrm{mM}$ sodium pyruvat and penicillin/streptomicin. The cells were kept in culture at $37^{\circ} \mathrm{C}$ and $5 \% \mathrm{CO}_{2}$, and the medium was changed every 3 days. To keep the cells at optimal proliferating conditions, they were passaged at $80 \%$ confluence and seeded at $20 \%$ confluence.

siRNA transient transfection experiments. Twenty-four hours before each transfection experiment, $9 \times 10^{4}$ cells $(30 \%$ confluence) were seeded in a 6-well plate in DMEM without antibiotics. The transfections were performed using Lipofectamine 2000 transfection reagent (Invitrogen) according to the manufacturer's instructions for a final 1:2 or 1:3 ratio. After transfection, cells were cultivated in standard conditions, collected at time points of 40 and $80 \mathrm{~h}$, and harvested in lysis buffer or Trizol reagent (Gibco BRL) for TRAP assay and semi-quantitative (sq)RT-PCR, respectively. As a control, the GFP-22 siRNA was used with the same transfection conditions.

Stable transfections. The molecules that produced the best inhibition effect in transient experiments (siRNA \#1) were then selected for long-term inhibition analyses. The siRNA \#1 sequence was cloned as a short hairpin (sh)RNA into the pSilencerTH 3.1-H1 neo expression vector (Ambion), generating pSilencer si1-H1-neo. The vector was amplified and sequenced to exclude possible mutations at the shRNA sequence and $\mathrm{H} 1$ promoter regions.

For the stable transfection, cells were seeded in a 6-well plate for a final $15 \%$ confluence. Twenty-four hours after seeding, $0.5 \mu \mathrm{g}$ plasmid-DNA was mixed with Effectene transfection reagent (Qiagen) according to the manufacturer's instructions for a final 1:10 ratio. Antibiotic selection with G418 was started $24 \mathrm{~h}$ after transfection and completed in 2 weeks. Cells were then plated at low density and six clones were isolated and expanded in culture. After clone expansion, cell aliquots were collected for the analysis of hTERT mRNA expression by sqRT-PCR and telomerase activity by TRAP assay. As controls, the original non-transfected cell line and one clone transfected with a non-sense shRNA were analyzed accordingly. The vector carrying the non-sense shRNA sequence (5'-ACTACCGTTGTTAAGGTG-3') was supplied with the pSilencerTH 3.1-H1 Neo Expression Vector kit (Ambion).

Telomerase activity. TRAP assay (telomeric repeat amplification protocol) was performed as described previously (14).

Quantification of hTERT $m R N A$. For the sqRT-PCR analysis, total RNA was isolated using Trizol reagent (Sigma) and reverse transcribed using random hexamers with Superscript II (Invitrogen). For hTERT mRNA detection, cDNA was mixed with the PCR reaction mixture and each hTERT-specific primer (LT5 and LT6). The PCR cycle number was set up to permit the distinction between mRNA expression profiles among the samples. PCR products were visualized on $1.2 \%$ agarose gel. $\beta$-actin was used as a normalizing internal control, and positive and negative controls were always included. PCR reagents were from the Master Taq kit (Eppendorf).

Telomere length measurement. Overall telomere length measurement by Southern blot analysis was performed as described (15). In short, genomic DNA was extracted from cell cultures according to standard protocols. DNA $(5 \mu \mathrm{g})$ 
SiRNA \#1

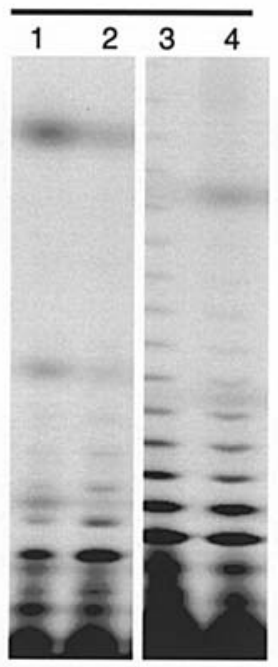

SiRNA \#2

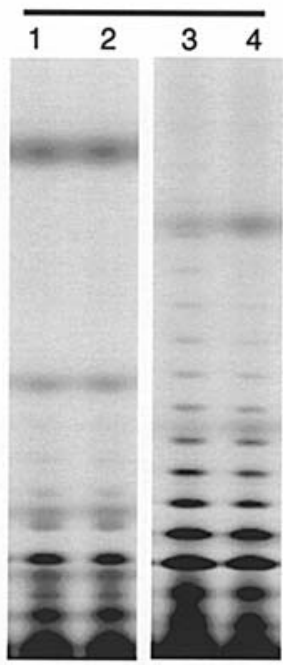

SiRNA \#3

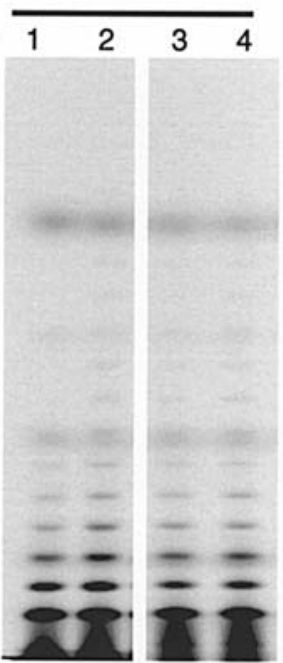

Control

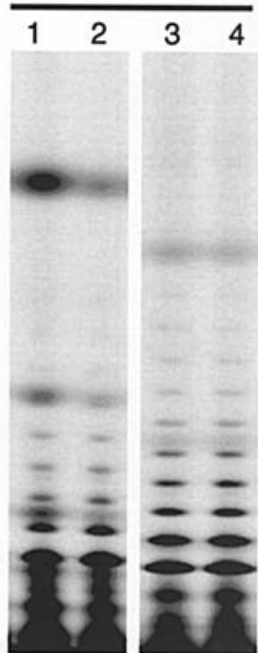

Figure 1. Telomerase transient inhibition in the HT29 cell line. Cells were transfected with siRNA \#1, siRNA \#2, siRNA \#3 or control GFP siRNA, as indicated. Lipofectamine 2000 was used in a 1:2 or 1:3 final ratio and cells were collected after 40-h (lanes 1 and 2) and 80-h (lanes 3 and 4) incubation. Among the three selected siRNAs, the siRNA \#1 molecule produced the best inhibition after 40-h incubation, and telomerase activity was restored after 80 h.

was digested with Hinfl restriction enzyme and fractionated in $0.8 \%$ agarose gel. The transfer was carried out in 20X SSC buffer overnight at room temperature. The membrane was UV-crosslinked, washed in 2X SSC and air dried. Southern blot hybridization was performed using a DIG system (Roche) according to the manufacturer's instructions with minimal modifications. The 5' digoxigenin-labeled oligo-(TTAGGG) (Roche) was used as a telomere-specific hybridization probe.

MSI analysis. Markers BAT25, BAT26, D2S123, D5S346 and D17S250, established for the detection of microsatellite instability (16), were used for MSI analysis. We compared genomic DNA stability at those five regions in the stable transfected clones (clone \#5 and the non-sense clone) to the profile of the corresponding untransfected HT29 cell line. Cells were analyzed at passages 2 and 20. Genomic DNA was extracted using standard protocols and subjected to PCR amplification. PCR conditions were previously established and routinely applied in our laboratory (17).

\section{Results}

Identification of a telomerase inhibitory siRNA by transfection of HT29 cells. To select the siRNA molecule that produces the most efficient telomerase inhibition, the HT29 cell line was transiently transfected with three different 21 nt doublestranded siRNAs directed against specific regions of the hTERT mRNA. siRNA \#1 targets the exon/exon splicing boundary of exons 10 and 11, siRNA \#2 was directed against a region on exon 4 and siRNA \#3 was directed against the $3^{\prime}$ portion of exon 11 .

As revealed by TRAP assay, a significant reduction in telomerase activity was achieved with siRNA \#1 when the three siRNA molecules were compared with the GFP control experiment (Fig. 1). However, as expected, this effect was transient in duration and telomerase activity was restored $80 \mathrm{~h}$ after siRNA transfection.
Thus, for the long-term analysis of the telomerase inhibition effects, the siRNA \#1 sequence was cloned as an shRNA into the pSilencer 3.1-H1 neo eukaryotic expression vector (Ambion) generating the pSilencer si1-H1-neo vector. The HT29 cell line was then stably transfected with that construct.

Establishment of a stable telomerase knockdown cell lineage. To investigate the consequences of telomerase long-term inhibition, the HT29 cell line was transfected with the cloned pSilencer si1-H1-neo that stably expressed an shRNA sequence previously selected in the transient transfection experiments. The G418 selection step was completed in two weeks, and the remaining transfectants were then plated at a low density. Six cell clones were isolated and propagated in culture. Aliquots of the cells were tested for telomerase inhibition with TRAP assay and the expression of mRNA was tested by sqRT-PCR. One of the isolated clones displayed high levels of telomerase inhibition. Clone \#5 was then kept in culture for further analysis, together with the non-sense clone and non-transfected cell line.

The question of whether clone \#5 retains its telomerasenegative phenotype among the subsequent divisions was analyzed with TRAP assay and sqRT-PCR at three different passages. As observed in Fig. 2 at the three passages analyzed, telomerase-negative phenotype and hTERT mRNA knockdown were retained from the first passage in culture (passage 2) until the end of proliferation (passage 22). To further confirm the stability of the transfection, an aliquot of clone \#5 frozen at passage 2 was reintroduced into cell culture and subcloned. After 3 weeks propagation, the three subclones were tested with TRAP assay and sqRT-PCR, and showed neither telomerase activity nor hTERT mRNA expression in comparison with the reintroduced non-sense control and non-transfected cell line. As a complement, genomic DNA extracted from clone \#5 and non-sense control were subjected to PCR reaction for plasmid-DNA detection, with a primer set that specifically anneals at vector sequences. The analysis revealed that clone 
(A)

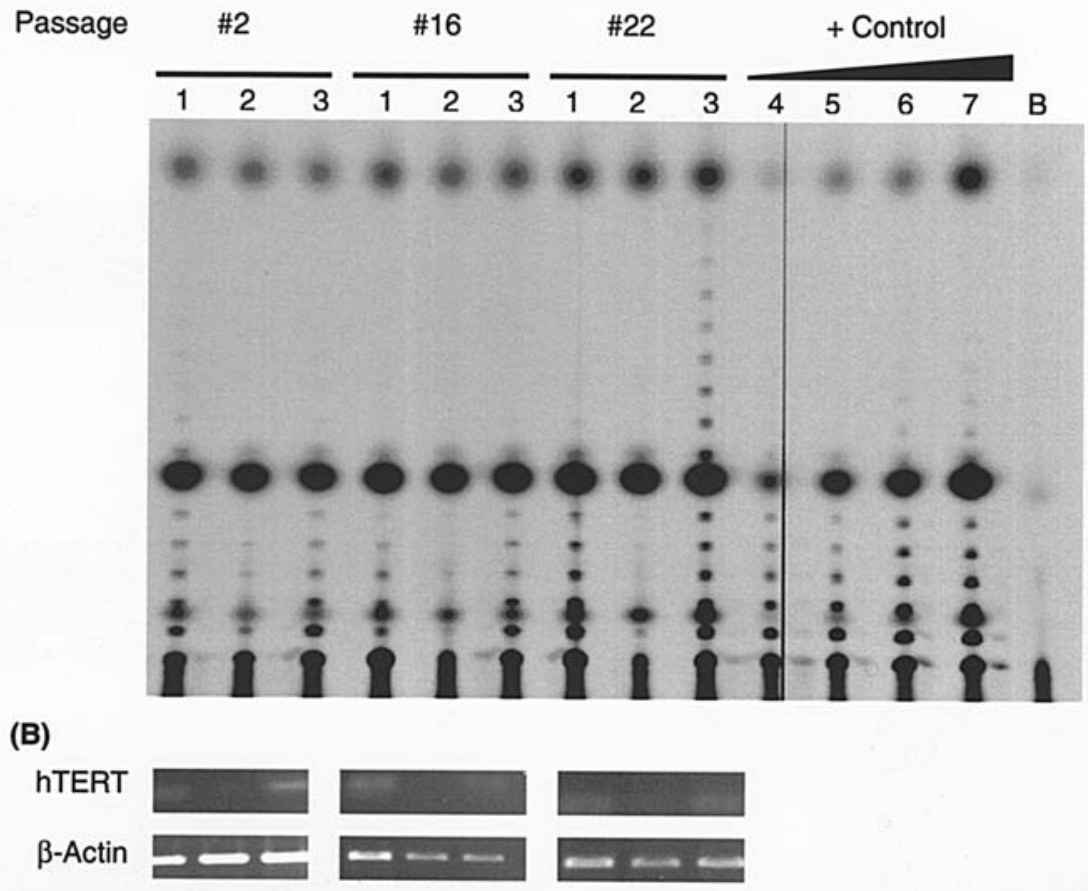

Figure 2. TRAP assay (A) and sqRT-PCR (B) analysis in HT29 stably transfected cells after subsequent passages. Cells were analyzed with TRAP assay and sqRT-PCR for telomerase activity and hTERT mRNA expression, respectively, at the indicated passage numbers. Lane 1, non-sense control; lane 2, clone \#5; and lane 3, non-transfected cell line. Lanes 4, 5, 6 and 7 correspond to 0.1, 0.25, 0.5 and $1.0 \mu \mathrm{g}$ of serial protein dilutions from an HT29 control sample for demonstration of the semi-quantitative approach. For sqRT-PCR, B-actin was used as a normalizing internal control. B, blank.

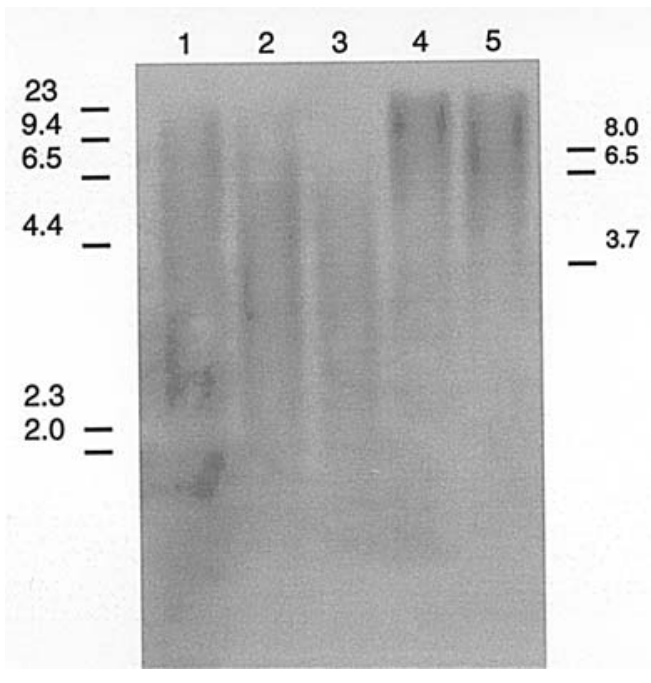

Figure 3. Overall telomere length measurement in stably transfected HT29 clone \#5 and non-sense control after 2,10 and 20 passages. Genomic DNA was digested with HinfI and blotted to the membrane. Telomerase probe $\left(5^{\prime}{ }^{\prime} \text { CCCTAA } 3 '\right)_{3}$ was $5^{\prime}$ tagged with digoxigenin. Lanes 1,2 and 3 , clone \#5 after 2, 10 and 20 passages, respectively; lanes 4 and 5, non-sense control and non-transfected cell line, respectively, both at passage 20 . The overall telomere length in clone \#5 after 20 PDs was markedly reduced in comparison with the corresponding controls. The $\lambda$ DNA-HindIII (left) and the $\lambda$ DNAEco47I markers (right) were expressed in $\mathrm{kb}$.

\#5 and the non-sense control carry the respectively cloned vectors stably integrated into their genomes (data not shown). These results confirm the stability of the telomerase-negative phenotype after transfection with the pSilencer si1-H1-neo cloned vector.

MSI analysis. Considering that the original HT29 cells have no MSI instability and no mismatch repair deficiency (27), we investigated whether telomerase inhibition with our shRNA could lead to MSI instability. Genomic DNA isolated from clone \#5 and non-sense control aliquots at passages 2 and 20 were subjected to PCR reaction for amplification of a panel of five microsatellite markers. As a control, the nontransfected cell line was used. Results showed that no MSI instability was found in the five loci analyzed at both passages when clone \#5 was compared with the corresponding controls (data not shown). Thus, telomerase inhibition in clone \#5 did not affect microsatellite stability in the five markers analyzed.

Overall telomere length measurement. As telomere attrition could be a direct consequence of telomerase inhibition, we investigated the overall telomere length in clone \#5 after telomerase inhibition. Genomic DNA isolated from clone \#5 aliquots at passages 2, 10 and 20 was compared to the nonsense control and non-transfected HT29 cell line, both at passage 20, by Southern hybridization analysis. As seen in Fig. 3, telomere length gradually decreased among the subsequent cell divisions, but no telomere attrition was observed in the controls after 20 passages in culture. This result was in accordance with the telomerase-negative phenotype observed in clone \#5 and indicated that telomerase was efficiently inhibited by our shRNA sequence leading to telomere attrition. After 22 population doublings (approx- 
imately 4 months), cell clone \#5 stopped proliferating and the cells died.

\section{Discussion}

With the described approach, we were able to inhibit in vitro telomerase activity in the HT29 colorectal immortal cell line using an shRNA vector-based strategy for a considerable number of cell divisions. RNA interference is known to be variable in effect when applied to different regions of a gene. Thus, we were not surprised when only 1 of 3 different tested siRNAs, all directed against distinct regions onto the hTERT gene, displayed telomerase inhibition. Only in isolated cell clone \#5, obtained by stable transfection with our designed siRNA molecule \#1 and directed against the splice junction of exons 10 and 11 of hTERT mRNA, was telomerase activity blocked due to down-regulation of expressed hTERT. The cells of clone \#5 do not show morphological alterations compared to the control cells that were concomitantly cultivated, but there are obvious differences in the inhibitory effect. The observation that 5 of 6 isolated clones displayed a telomerase-positive phenotype could be due to the integration site of the expression construct into the cellular genome or cellular resistance mechanisms such as methylation inactivation of the transgene (18).

Telomerase activity is strongly associated with telomere length maintenance. Due to the incomplete replication of the lagging strand at the end of each replication cycle, telomeres become shorter (19). For 22 population doublings, cell clone \#5 retained the telomerase-negative phenotype and was subsequently accompanied by a dramatic telomere attrition. The telomerase-negative cells then stopped further proliferation, possibly reaching a state called replicative senescence that is presumably induced by cellular control mechanisms initiated by too short telomeres (20). Here, apoptosis cannot be controlled by activated wild-type p53, as the HT29 cell line is characterized by an Arg273His substitution caused by a p53 gene mutation commonly found in colon cancer. This dominant-negative mutation is responsible for the loss of the DNA-binding capacity of p53 homo- or heterotetramers (12).

Cellular aging may be one consequence of telomere attrition. In normal telomerase-negative cells, telomere shortening may function as a mitotic clock that counts how many cellular divisions a cell can undergo before achieving replicative senescence. It was already observed that one feature of aging is the appearance of microsatellite instability. Previous studies showed that peripheral blood cells from old donors have an elevated frequency of microsatellite instability that could not be associated with defects in mismatch repair genes $(21,22)$. Additionally, some evidence indicates that telomerase reactivation in cancer cells provides other advantages not related to telomere stabilization, which are involved in increased repair system efficiency (23) and augmented chromosomal stability (24).

Based on these studies and considering that the HT29 cell line has no MSI instability and no mismatch repair deficiency (13), we questioned whether telomerase inhibition by our shRNA may lead to MSI. Both genetic instability, which is frequently induced by dysfunctional shortened telomeres, and replicative senescence (25) were not detectable despite the defective telomere homeostasis. In HT29 telomerase-inactive cell clone \#5, there was no switch to an alternative lengthening of the telomere pathway (ALT), which was found in some telomerase-negative immortal cell lines (26). Interestingly, telomeric recombination in mismatch repair-deficient human colon cancer cells after telomerase inhibition was already described (27). The mismatch repair system of cell clone \#5 appeared to still be intact as shown with the five MSI loci examined after 20 population doublings of cell clone \#5. Thus, telomere erosion seems to be independent of microsatellite instability in HT29 cells.

It was reported that siRNA against hTR is more effective than inhibition of hTERT (28). However, we hypothesized that the inhibition of telomerase via hTERT-directed siRNA is more specific at least in colorectal tumorigenesis than hTR, which is also present in normal mucosa cells (29). Moreover, plasmids as a DNA vector for transfection seem to be more secure retroviral vectors concerning future therapeutic application despite being less efficient than a retroviral system. Therefore, our described approach of telomerase silencing using siRNA \#1 as a potent telomerase inhibitor could be relevant for further applications in cancer therapy.

Furthermore, the enlightenment of pathways involved in telomerase-dependent regulation is a fundamental aspect of cell cycle control in tumorigenesis and could provide new insights into the roles of telomerase in processes such as immortalization and carcinogenesis of colonocytic cells. There is increasing evidence that telomerase has other roles in addition to the stabilization of telomeres in cells (30). It was suggested that cancer cells are 'telomerase addicted' (31). Using comparative global expression profiling between original HT29 cells and telomerase-inactivated cell clones in different passages, the established cell clone \#5 should be qualified to uncover the functions of telomerase in tumor growth and progression and telomere maintenance.

\section{Acknowledgements}

Supported by the DAAD (P.N.) and the 'Wilhelm-Roux Programm' (FKZ 4/40).

\section{References}

1. Morin GB: The human telomere terminal transferase enzyme is a ribonucleoprotein that synthesizes TTAGGG repeats. Cell 59: 521-529, 1989.

2. Kim NW, Piatyszek MA, Prowse KR, Harley CB, West MD, Ho PL, Coviello GM, Wright WE, Weinrich SL and Shay JW: Specific association of human telomerase activity with immortal cells and cancer. Science 266: 2011-2015, 1994.

3. Naito $Y$, Takagi $T$, Handa O, Ishikawa $T$, Matsumoto $N$, Yoshida N, Kato H, Ando T, Takemura T, Itani K, Hisatomi H, Tsuchihashi $\mathrm{Y}$ and Yoshikawa T: Telomerase activity and expression of telomerase RNA component and catalytic subunits in precancerous and cancerous colorectal lesions. Tumour Biol 22: 374-382, 2001.

4. Guittat L, Alberti P, Gomez D, De Cian A, Pennarun G, Lemarteleur T, Belmokhtar C, Paterski R, Morjani H, Trentesaux C, Mandine E, Boussin F, Mailliet P, Lacroix L, Riou JF and Mergny JL: Targeting human telomerase for cancer therapeutics. Cytotechnology 45: 75-90, 2004.

5. Saretzki G: Telomerase inhibition as cancer therapy. Cancer Lett 194: 209-219, 2003.

6. Brown T, Sigurdson E, Rogatko A and Broccoli D: Telomerase inhibition using azidothymidine in the HT-29 colon cancer cell line. Ann Surg Oncol 10: 910-915, 2003. 
7. Hannon GJ: RNA interference. Nature 418: 244-251, 2002.

8. Elbashir SM, Lendeckel W and Tuschl T: RNA interference is mediated by 21 - and 22-nucleotide RNAs. Genes Dev 15: 188-200, 2001.

9. Nakamura M, Masutomi K, Kyo S, Hashimoto M, Maida Y, Kanaya T, Tanaka M, Hahn WC and Inoue M: Efficient inhibition of human telomerase reverse transcriptase expression by RNA interference sensitises cancer cells to ionising radiation and chemotherapy. Hum Gene Ther 16: 859-868, 2005.

10. Li S, Rosenberg JE, Donjacour AA, Botchkina IL, Hom YK, Cunha GR and Blackburn EH: Rapid inhibition of cancer cell growth induced by lentiviral delivery and expression of mutanttemplate telomerase RNA and anti-telomerase short-interfering RNA. Cancer Res 64: 4833-4840, 2004.

11. Sciamanna I, Landriscina M, Pittoggi C, Quirino M, Mearelli C, Beraldi R, Mattei E, Serafino A, Cassano A, Garaci E, Sinibaldi-Vallebona P, Barone C and Spadafora C: Inhibition of endogenous reverse transcriptase antagonizes human tumor growth. Oncogene 24: 3923-3931, 2005.

12. De Vries A, Flores ER, Miranda B, Hsieh HM, van Oostrom CT, Sage J and Jacks T: Targeted point mutations of p53 lead to dominant-negative inhibition of wild-type p53 function. Proc Natl Acad Sci USA 99: 2948-2953, 2002.

13. Boldog F, Gemmill RM, West J, Robinson M, Robinson L, Li E, Roche J, Todd S, Waggoner B, Lundstrom R, Jacobson J, Mullokandov MR, Klinger H and Drabkin HA: Chromosome 3 p14 homozygous deletions and sequence analysis of FRA3B. Hum Mol Genet 6: 193-203, 1997.

14. Fiedler W, Junker K, Schlichter A, Schubert J, Ernst G, Dahse R and Claussen U: Determination of telomerase activity for differential analysis of multifocal renal cell carcinomas. Kidney Int 56: 1286-1288, 1999.

15. Dahse R, Fiedler W, Ernst G, Kosmehl H, Schlichter A, Schubert $\mathbf{J}$ and Claussen U: Changes in telomere lengths in renal cell carcinomas. Cell Mol Biol 42: 477-484, 1996.

16. Boland CR, Thibodeau SN, Hamilton SR, Sidransky D, Eshleman JR, Burt RW, Meltzer SJ, Rodriguez-Bigas MA, Fodde R, Ranzani GN and Srivastava S: A National Cancer Institute Workshop on Microsatellite Instability for cancer detection and familial predisposition: development of international criteria for the determination of microsatellite instability in colorectal cancer. Cancer Res 58: 5248-5257, 1998.

17. Koch E, Fiedler W, Tannapfel A and Ballhausen WG: Alteration of the fragile histidine triad gene in intrahepatic cholangiocarcinoma. Eur J Gastroenterol Hepatol 15: 907-913, 2003.

18. Chen ZY, He CY, Meuse L and Kay MA: Silencing of episomal transgene expression by plasmid bacterial DNA elements in vivo. Gene Ther 11: 856-864, 2004.
19. Ohki R, Tsurimoto $\mathrm{T}$ and Ishikawa $\mathrm{F}$ : In vitro reconstitution of the end replication problem. Mol Cell Biol 21: 5753-5766, 2001.

20. Artandi SE and Attardi LD: Pathways connecting telomeres and p53 in senescence, apoptosis, and cancer. Biochem Biophys Res Commun 331: 881-890, 2005.

21. Krichevsky S, Pawelec G, Gural A, Effros RB, Globerson A, Yehuda DB and Yehuda AB: Age related microsatellite instability in T cells from healthy individuals. Exp Gerontol 39: 507-515, 2004.

22. Coolbaugh-Murphy MI, Xu J, Ramagli LS, Brown BW and Siciliano MJ: Microsatellite instability (MSI) increases with age in normal somatic cells. Mech Ageing Dev 126: 1051-1059, 2005.

23. Sharma GG, Gupta A, Wang H, Scherthan H, Dhar S, Gandhi V, Iliakis G, Shay JW, Young CS and Pandita TK: hTERT associates with human telomeres and enhances genomic stability and DNA repair. Oncogene 22: 131-146, 2003.

24. Pirzio LM, Freulet-Marrière M-A, Bai Y, Fouladi B, Murnane JP, Sabatier L and Desmaze C: Human fibroblsts expressing hTERT show remarkable karyotype stability even after exposure to ionizing radiation. Cytogenet Genome Res 104: 87-94, 2004.

25. Hackett JA and Greider CW: End resection initiates genomic instability in the absence of telomerase. Mol Cell Biol 23: 8450-8461, 2003

26. Stewart SA: Telomere maintenance and tumorigenesis: an 'ALT'ernative road. Curr Mol Med 5: 253-257, 2005.

27. Bechter OE, Zou Y, Walker W, Wright WE and Shay JW: Telomeric recombination in mismatch repair deficient human colon cancer cells after telomerase inhibition. Cancer Res 64 3444-3451, 2004.

28. Natarajan S, Chen Z, Wancewicz EW, Monia BP and Corey DR: Telomerase reverse transcriptase (hTERT) mRNA and telomerase RNA (hTR) as targets for downregulation of telomerase activity. Oligonucleotides 14: 263-273, 2004.

29. Yan P, Saraga EP, Bouzourene H, Bosman FT and Benhattar J. Expression of telomerase genes correlates with telomerase activity in human colorectal carcinogenesis. J Pathol 193: 21-26, 2001.

30. Sarin KY, Cheung P, Gilison D, Lee E, Tennen RI, Wang E, Artandi MK, Oro AE and Artandi SE: Conditional telomerase induction causes proliferation of hair follicle stem cells. Nature 436: 1048-1052, 2005.

31. Li S, Crothers J, Haqq CM and Blackburn EH: Cellular and gene expression responses involved in the rapid growth inhibition of human cancer cells by RNA interference-mediated depletion of telomerase RNA. J Biol Chem 280: 23709-23717, 2005. 